\title{
CAD of Complex Passive Devices Composed of Arbitrarily Shaped Waveguides Using Nyström and BI-RME Methods
}

\author{
Máriam Taroncher, Student Member, IEEE, Ana Vidal, Member, IEEE, Vicente E. Boria-Esbert, Senior Member, IEEE, \\ Stephan Marini, Santiago Cogollos, Jordi Gil, and Benito Gimeno, Member, IEEE
}

\begin{abstract}
In this paper, a novel computer-aided design (CAD) tool of complex passive microwave devices in waveguide technology is proposed. Such a tool is based on a very efficient integral-equation analysis technique that provides a full-wave characterization of discontinuities between arbitrarily shaped waveguides defined by linear, circular, and/or elliptical arcs. For solving the modal analysis of such arbitrary waveguides, a modified version of the well-known boundary integral-resonant-mode expansion (BI-RME) method using the Nyström approach, instead of the traditional Galerkin version of the method of moments, is proposed, thus providing significant savings on computational costs and implementation complexity. The novel theoretical aspects of this Nyström approach, as well as their impact on the original BI-RME formulation, are fully described. Comparative benchmarks between this new technique and the classical BI-RME formulation using Galerkin are successfully presented for the full-wave analysis of frequently used irises (i.e., rectangular cross-shaped and circular multiridged) and for the CAD of complex waveguide components (such as rectangular waveguide filters considering mechanization effects and dual-mode circular waveguide filters with elliptical irises).
\end{abstract}

Index Terms-Integral equations (IEs), method of moments (MoM), waveguide components, waveguide discontinuities.

\section{INTRODUCTION}

A RBITRARILY shaped waveguides, whose cross sections are defined by a combination of linear, circular, and/or elliptical waveguides, are increasingly used in passive waveguide components (e.g., filters, diplexers and multiplexers, directional couplers, power dividers and combiners, orthomode transducers, polarizers, twisters, and mode launchers) [1]. Most available computer-aided design (CAD) tools used for such devices are based on numerical meshing techniques, i.e., the finite-element (FE) method [2], the finite-difference time-domain (FDTD) method [3], and the transmission-line

Manuscript received October 1, 2004; revised February 28, 2005. This work was supported by the Ministerio de Ciencia y Tecnología, Spanish Government, under the Special Action of Space National Plan ESP2001-4547-PE.

M. Taroncher, A. Vidal, V. E. Boria-Esbert, S. Marini, S. Cogollos, and J. Gil are with the Departamento de Comunicaciones, Escuela Téchnica Superior de Ingenieros Telecomunicación, Universidad Politécnica de Valencia, E-46022 Valencia, Spain (e-mail: avidal@dcom.upv.es; vboria@dcom.upv.es).

B. Gimeno is with the Departamento Física Aplicada-Instituto de Ciencias de los Materiales de la Universtat de Valencia, Universidad de Valencia, E-46100 Burjassot, Valencia, Spain.

Digital Object Identifier 10.1109/TMTT.2005.848795 matrix (TLM) method [4], which do have strong requirements on CPU time and memory storage. To alleviate these problems, several modal methods, such as those based on the generalized scattering matrix (GSM), generalized admittance matrix (GAM), or generalized impedance matrix (GIM) have been successfully proposed [5].

The above-mentioned modal methods do always require to know the complete modal chart (cutoff frequencies) of the involved arbitrarily shaped waveguides, as well as the coupling integrals between the modal vectors of such cascaded waveguides. Among the many different approaches proposed in the technical literature for the modal chart computation, the wellknown boundary integral-resonant-mode expansion (BI-RME) method has revealed to provide very accurate results in short computation times [6]. Recently, this method has been revisited in order to cope with arbitrary profiles defined by the combination of linear, circular, and/or elliptical waveguides [7]. Another advantage of the BI-RME technique is that, without hardly any additional CPU effort, the coupling coefficients between the arbitrarily shaped waveguide and a standard rectangular contour enclosing the arbitrary profile can be computed (see [8] and [9]).

Both practical BI-RME implementations described in [6] and [7] are based on the Galerkin version of the method of moments (MoM) [10], where the basis and testing functions are chosen to be overlapping piecewise parabolic splines. Such a choice does obviously introduce additional complexities to the practical solution of the BI-RME integral equations (IEs): first, the contribution of each parabolic piece to several matrix entries must be carefully accounted for (see [6]) and, secondly, the connection of different types of segments (linear, circular, and elliptical ones) must be treated as described in [7]. Furthermore, following the Galerkin approach, the regular terms of the BI-RME matrix elements become single and double integrals that can be solved numerically via a Gauss quadrature rule (see [7]), which can require a large number of integrand evaluations for high-accuracy applications. An alternative way to solve the cited IEs, thus avoiding the previous restrictions related to the Galerkin approach, is the so-called Nyström method described in [11]. This solution is much simpler than the Galerkin scheme since each matrix entry in the eigenvalue problem does only involve a function evaluation. However, the main disadvantage is that the Nyström method tends to increase the singularity problems that also arise with Galerkin. 


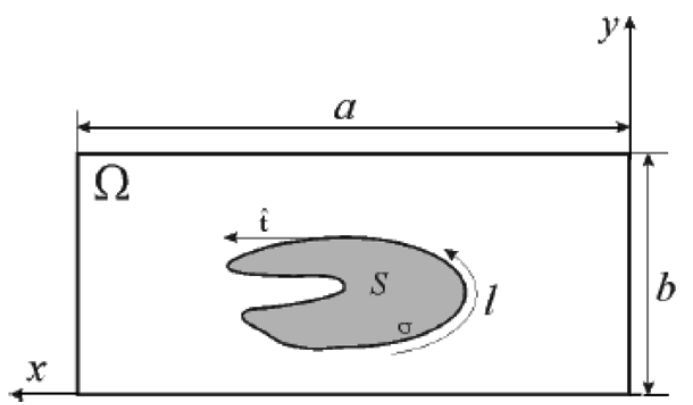

Fig. 1. Arbitrarily shaped waveguide (with cross section $S$ ) to be analyzed by the combination of Nyström and BI-RME methods.

The Nyström method is a simple and efficient point-based segmentation solution for solving IEs, where integrals are approximated by weighted sums of function evaluations

$$
\int f(x) d s=\sum_{j=1}^{N} q_{j} f\left(x_{j}\right) .
$$

When IEs involve singular kernels [11], it is necessary to locally adapt the quadrature weights $q_{j}$ to the singularity regions [12], [13]. In this study, the new formulation leads to singular and hyper-singular operators (TE case of the BI-RME formulation) that are specifically treated. The authors have firstly proposed the application of the Nyström and BI-RME methods to the modal chart determination of arbitrarily shaped waveguides in [14] and [15] where no theoretical details about the hyper-singularity treatment of the TE case are explicitly given.

This paper describes the fast and simple implementation of the Nyström-based BI-RME method for the accurate analysis of passive devices composed of waveguides with arbitrary contours (defined by linear, circular, and/or elliptical arcs). The integration of such a method within a CAD tool of complex passive devices, which is based on the characterization of waveguide discontinuities by means of an IE technique described in [16], is also discussed in this paper. Making use of the novel Nyström approach proposed, the complete modal charts of a rectangular cross-shaped iris and of a multiridged circular waveguide have been successfully computed. The more efficient CAD tool developed has been used in the accurate design of an inductively coupled rectangular waveguide filter considering rounded corners, and a dual-mode filter in a circular waveguide with elliptical irises and triangular tuning elements. The gain in efficiency while preserving accuracy due to the Nyström approach has been successfully measured by comparing our results with those provided by the Galerkin solution.

\section{NYSTRÖM FORMULATION}

Here, the formulation related to the combination of the Nyström and BI-RME methods is fully described. The arbitrarily shaped waveguide to be considered has a cross section defined by a combination of linear, circular, and/or elliptical arcs (see $S$ in Fig. 1). The arbitrary contour $\sigma$ of such a waveguide (defined by the tangent vector $\widehat{\mathbf{t}}$ and a suitable abscisa $l$, also shown in
Fig. 1) must be completely enclosed within a standard rectangular waveguide of cross section $\Omega$.

To characterize the arbitrary waveguides under consideration, we will only present the novel theoretical aspects related to the application of the Nyström method. Special emphasis will be given to the suitable treatment of the singular integrals that appear in some BI-RME matrix elements.

\section{A. TM Modes}

The longitudinal component of the electric field at a generic observation point $\mathbf{r}$ inside $S$ (see Fig. 1) may be represented as follows (cf. (7) in [6]):

$$
\begin{aligned}
& E_{z}(\mathbf{r})=-j \eta k^{\prime} \int_{\sigma} g\left(\mathbf{r}, \mathbf{s}^{\prime}\right) J_{z}\left(l^{\prime}\right) d l^{\prime} \\
& \quad-j \eta k^{\prime 3} \sum_{m=1}^{M^{\prime}} \frac{\psi_{m}^{\mathrm{TM} \square}(\mathbf{r})}{h_{m}^{\prime 2}\left(h_{m}^{\prime 2}-k^{\prime 2}\right)} \int_{\sigma} \psi_{m}^{\mathrm{TM} \square}\left(\mathbf{s}^{\prime}\right) J_{z}\left(l^{\prime}\right) d l^{\prime}
\end{aligned}
$$

where $g\left(\mathbf{r}, \mathbf{s}^{\prime}\right)$ is the rapidly convergent scalar two-dimensional (2-D) Green's function and $J_{z}\left(l^{\prime}\right)$ is the longitudinal component of the current density $\mathbf{J}_{\sigma}$. Moreover, $h_{m}^{\prime}$ and $\psi_{m}^{\mathrm{TM} \square}$ are, respectively, the cutoff wavenumber and normalized scalar potential of the $m$ th TM mode of the surrounding standard rectangular waveguide ( $\Omega$ in Fig. 1).

To compute the TM modes, the boundary condition for the axial component of the electric field $\left(E_{z}=0\right)$ is first imposed on $\sigma$. The arbitrary contour $\sigma$ is then segmented following the Nyström method, where the central point $\mathbf{s}_{j}$ and the length of a segment $\omega_{j}$ are only needed. With the Nyström approach, the integral is approximated at each point by a simple one-point trapezoidal quadrature, being the quadrature weights equal to the length of the segments of the discretization $\omega_{j}$, as described in [17]. The scalar Green function $g\left(\mathbf{s}_{i}, \mathbf{s}_{j}\right)$ is singular when the discrete source $\left(\mathbf{s}_{j}\right)$ and observation points $\left(\mathbf{s}_{i}\right)$ coincide. In such a case, the contribution of the segment $\sigma_{i}$ that produces the singularity is isolated and treated separately. The following system of equations is then obtained:

$$
\begin{aligned}
\sum_{\substack{j=1 \\
j \neq i}}^{N} \omega_{j} g\left(\mathbf{s}_{i}, \mathbf{s}_{j}\right) J_{z}\left(l_{j}\right)+\int_{\sigma_{i}} g\left(\mathbf{s}_{i}, \mathbf{s}^{\prime}\right) J_{z}\left(l^{\prime}\right) d l^{\prime} \\
+\sum_{m=1}^{M^{\prime}} \frac{\psi_{m}^{\mathrm{TM} \square}\left(\mathbf{s}_{i}\right)}{h_{m}^{\prime 2}} a_{m}^{\prime}=0 \\
\quad\left(1-\frac{k^{\prime 2}}{h_{m}^{\prime 2}}\right) \cdot a_{m}^{\prime}=k^{\prime 2} \sum_{j=1}^{N} \omega_{j} \frac{\psi_{m}^{\mathrm{TM} \square}\left(\mathbf{s}_{j}\right)}{h_{m}^{\prime 2}} J_{z}\left(\mathbf{s}_{j}\right)
\end{aligned}
$$

where $N$ is the number of points used for the segmentation of the perturbed contour.

Equations (3) and (4) lead to an eigenvalue problem similar to the one obtained with the original Galerkin-based BI-RME formulation, which can be expressed in matrix form as

$$
\left\{\left[\begin{array}{ll}
\mathbf{U} & \mathbf{O}_{\mathbf{t}} \\
\mathbf{O} & \mathbf{O}^{\prime}
\end{array}\right]-k^{\prime 2}\left[\begin{array}{cc}
\mathbf{D}^{\prime} & \mathbf{R}_{\mathbf{t}}^{\prime} \\
\mathbf{R}^{\prime} & \mathbf{L}^{\prime}
\end{array}\right]\right\}\left[\begin{array}{l}
\mathbf{a}^{\prime} \\
\mathbf{b}^{\prime}
\end{array}\right]=0
$$


where $\mathbf{U}$ is the $M^{\prime} \times M^{\prime}$ unitary matrix, $\mathbf{O}$ is the $N \times M^{\prime}$ null matrix, and $\mathbf{O}^{\prime}$ is the $N \times N$ null matrix. The detailed expressions for the other matrices are

$$
\begin{aligned}
\mathbf{D}^{\prime} & =\operatorname{diag}\left(h_{1}^{\prime-2}, h_{2}^{\prime-2}, \ldots, h_{M}^{\prime-2}\right) \\
L_{i j}^{\prime} & =g\left(\mathbf{s}_{i}, \mathbf{s}_{j}\right), \quad i \neq j ; \\
L_{i i}^{\prime}= & \frac{1}{\omega_{i}} \int_{\sigma_{i}} g\left(\mathbf{s}_{i}, \mathbf{s}^{\prime}\right) d l^{\prime} \\
R_{i m}^{\prime}= & \frac{\psi_{m}^{\mathrm{TM} \square}\left(\mathbf{s}_{i}\right)}{h_{m}^{\prime 2}}, \quad i, j=1,2, \ldots, N ; \\
& m=1,2, \ldots, M^{\prime} .
\end{aligned}
$$

It must be noticed that the expressions for the elements of the $L^{\prime}$ and $R^{\prime}$ matrices are different to the equivalent ones deduced for the Galerkin approach (cf. [6, eqs. (16b) and (16c)]), and their new computation is much more efficient and simpler.

In the generalized eigenvalue problem defined by (5), $\mathbf{a}^{\prime}$ is a column vector including the modal coefficients $a_{m}^{\prime}$, whereas $\mathbf{b}^{\prime}$ contains the terms $b_{j}^{\prime}=\omega_{j} J_{z}\left(\mathbf{s}_{j}\right)$, where the quadrature weights have been included to preserve the symmetry of the involved matrices. The solution of the eigenvalue problem $\left(k^{\prime}\right)$ are the TM cutoff wavenumbers of the arbitrary waveguide.

As it happens with the Galerkin solution of the BI-RME method, the previous TM generalized eigenvalue problem can also be cast into the following standard form:

$$
\left(\mathbf{D}^{\prime}-\mathbf{R}_{t}^{\prime} \mathbf{L}^{\prime-1} \mathbf{R}^{\prime}\right) \mathbf{a}^{\prime}=k^{\prime-2} \mathbf{a}^{\prime}
$$

Finally, we must focus on the accurate computation of the diagonal elements $\left(L_{i i}^{\prime}\right)$ of the $\mathbf{L}^{\prime}$ matrix, which must cope with the singularity due to the evaluation of the scalar Green function with coincident observation and source points. In this case, a similar procedure to the one described in [7] for the Galerkin approach will be followed. First, the original scalar 2-D Green function can be split into a regular $\left(g_{r}\right)$ and a singular expression. This singular expression is then regularized following the technique explained in [18], giving as a final result a regular part of the singular expression $g_{r s}$ and a final singular term $g_{s}$ that can be analytically integrated. Consequently, the following expression for the diagonal elements of the $\mathbf{L}^{\prime}$ matrix is obtained in our case:

$$
\begin{aligned}
L_{i i}^{\prime} & =\left[g_{r}\left(\mathbf{s}_{i}, \mathbf{s}_{i}\right)+g_{r s}\left(\mathbf{s}_{i}, \mathbf{s}_{i}\right)\right]+\frac{1}{\omega_{i}} \int_{\sigma_{i}} g_{s}\left(\mathbf{s}_{i}, \mathbf{s}^{\prime}\right) d l^{\prime} \\
& =L_{i i r}^{\prime}+L_{i i s}^{\prime}
\end{aligned}
$$

where the regular term $L_{\text {iir }}^{\prime}$ can be directly computed, and the singular integral $L_{i i s}^{\prime}$ must be analytically solved. It should be noted that the integral solution depends on the geometry of the segment $\sigma_{i}$ (linear, circular, or elliptical geometry), whose detailed expressions for each kind of arc is as follows.

1) Linear Arcs:

$$
L_{i i s}^{\prime}=-\frac{1}{2 \pi}\left[\ln \left(\frac{\omega_{i}}{2}\right)-1\right]
$$

2) Circular Arcs:

$$
L_{i i s}^{\prime}=-\frac{1}{2 \pi}\left[\ln \left(\frac{\omega_{i}}{2}\right)-1\right] .
$$

\section{3) Elliptical Arcs:}

$$
\begin{aligned}
& L_{i i s}^{\prime}=-\frac{1}{4 \pi} \ln \left(a^{2} \sin ^{2} \eta_{m i}+b^{2} \cos ^{2} \eta_{m i}\right) \\
&-\frac{\gamma}{4 \pi} \frac{\left|\Delta \eta_{i}\right| a}{\omega_{i}}\left[\ln \left(\frac{\left|\Delta \eta_{i}\right|}{2}\right)-1\right]
\end{aligned}
$$

where $\gamma=\sqrt{1-e^{2} \cos ^{2} \eta_{m i}}, \Delta \eta_{i}=\eta_{2 i}-\eta_{1 i}$ and $\eta_{m i}=$ $0.5\left(\eta_{1 i}+\eta_{2 i}\right)$, with $\eta_{1 i}$ and $\eta_{2 i}$ being the elliptical parametric initial and final angles for $\sigma_{i}$, as defined in [7].

If the arbitrary waveguide supports TEM modes, the Nyström approach just outlined can also be employed for solving such problem. Following the same procedure explained in [6], the TEM solution may be obtained from a linear system of equations that involves the $L^{\prime}$ matrix.

\section{B. TE Modes}

The transversal component of the electric field at a generic observation point $\mathbf{r}$ inside $S$ (see Fig. 1) may be represented as follows (cf. [6, eq. (6)]):

$$
\begin{aligned}
\mathbf{E}_{t}(\mathbf{r})= & j \frac{\eta}{k} \int_{\sigma} \nabla \frac{\partial g\left(\mathbf{r}, \mathbf{s}^{\prime}\right)}{\partial l^{\prime}} J_{t}\left(l^{\prime}\right) d l^{\prime}-j \eta k \int_{\sigma} \overline{\mathbf{G}}_{s t}\left(\mathbf{r}, \mathbf{s}^{\prime}\right) \\
& \cdot \widehat{\mathbf{t}}\left(l^{\prime}\right) J_{t}\left(l^{\prime}\right) d l^{\prime}-j \eta k^{3} \sum_{m=1}^{M} \frac{\mathbf{e}_{m}^{\mathrm{TE}}(\mathbf{r})}{h_{m}^{2}\left(h_{m}^{2}-k^{2}\right)} \int_{\sigma} \mathbf{e}_{m}^{\mathrm{TE}}\left(\mathbf{s}^{\prime}\right) \\
& \cdot \widehat{\mathbf{t}}\left(l^{\prime}\right) J_{t}\left(l^{\prime}\right) d l^{\prime}
\end{aligned}
$$

where $\overline{\mathbf{G}}_{s t}\left(\mathbf{r}, \mathbf{s}^{\prime}\right)$ is the solenoidal dyadic Green function and $J_{t}\left(l^{\prime}\right)$ is the transversal component of the unknown current density $\mathbf{J}_{\sigma}$. On the other hand, $h_{m}$ and $\mathbf{e}_{m}^{\mathrm{TE}} \mathrm{E}^{\square}$ are, respectively, the cutoff wavenumber and normalized transversal electric field of the $m$ th TE mode of the surrounding standard rectangular waveguide related to the BI-RME method.

To compute the TE modes, the boundary condition to the tangential component of the electric field $\left(\mathbf{E}_{t} \cdot \widehat{\mathbf{t}}=0\right)$ is first imposed on $\sigma$. It should be noticed that the evaluation of the first term of (14) requires the numerical computation of a double partial derivative of $g$ with respect to the observation and source contour parameters $l$ and $l^{\prime}$. The derivatives of the Green functions are usually avoided in the literature (see [13]) because they present stronger singularities than the original functions. However, the double derivative of the scalar Green function has been obtained and accelerated, as shown in Appendix I. Such double derivative presents a hypersingularity that has been treated via the traditional method of adding and subtracting an asymptotic term [11], [12]

$$
\int_{\sigma} \frac{\partial^{2} g\left(\mathbf{s}, \mathbf{s}^{\prime}\right)}{\partial l \partial l^{\prime}}\left[J_{t}\left(l^{\prime}\right)-J_{t}(l)\right] d l^{\prime}+J_{t}(l) \int_{\sigma} \frac{\partial^{2} g\left(\mathbf{s}, \mathbf{s}^{\prime}\right)}{\partial l \partial l^{\prime}} d l^{\prime}
$$


where it can be easily proven that the second term is equal to zero when the arbitrary geometry is composed of closed contours and/or arcs connected to the surrounding standard waveguide, which applies to the considered devices.

Once this treatment is employed in the application of the Nyström method, the following expressions are obtained:

$$
\begin{aligned}
& \frac{1}{k^{2}} \sum_{\substack{j=1 \\
j \neq i}}^{N} \omega_{j} \frac{\partial^{2} g\left(\mathbf{s}_{i}, \mathbf{s}_{j}\right)}{\partial l \partial l^{\prime}}\left[J_{t}\left(\mathbf{s}_{j}\right)-J_{t}\left(\mathbf{s}_{i}\right)\right] \\
& -\sum_{\substack{j=1 \\
j \neq i}}^{N} \omega_{j} \widehat{\mathbf{t}}\left(\mathbf{s}_{i}\right) \cdot \overline{\mathbf{G}}_{s t}\left(\mathbf{s}_{i}, \mathbf{s}_{j}\right) \cdot \widehat{\mathbf{t}}\left(\mathbf{s}_{j}\right) J_{t}\left(\mathbf{s}_{j}\right) \\
& -\int_{\sigma_{i}} \widehat{\mathbf{t}}\left(\mathbf{s}_{i}\right) \cdot \overline{\mathbf{G}}_{s t}\left(\mathbf{s}_{i}, \mathbf{s}^{\prime}\right) \cdot \widehat{\mathbf{t}}\left(l^{\prime}\right) J_{t}\left(l^{\prime}\right) d l^{\prime} \\
& -\sum_{m=1}^{M} \frac{\mathbf{e}_{m}^{\mathrm{TE} \square}\left(\mathbf{s}_{i}\right) \cdot \hat{\mathbf{t}}\left(\mathbf{s}_{i}\right)}{h_{m}^{2}} a_{m}=0 \\
& \left(1-\frac{k^{2}}{h_{m}^{2}}\right) \cdot a_{m}=k^{2} \sum_{j=1}^{N} \omega_{j} \frac{\mathbf{e}_{m}^{\mathrm{TE} \square}\left(\mathbf{s}_{j}\right) \cdot \widehat{\mathbf{t}}\left(\mathbf{s}_{j}\right)}{h_{m}^{2}} b_{j}
\end{aligned}
$$

which can be cast into matrix form as

$$
\left\{\left[\begin{array}{cc}
\mathbf{U} & \mathbf{O}_{\mathbf{t}} \\
\mathbf{O} & \mathbf{C}
\end{array}\right]-k^{2}\left[\begin{array}{cc}
\mathbf{D} & \mathbf{R}_{\mathbf{t}} \\
\mathbf{R} & \mathbf{L}
\end{array}\right]\right\}\left[\begin{array}{l}
\mathbf{a} \\
\mathbf{b}
\end{array}\right]=0
$$

where $\mathbf{U}$ is the $M \times M$ unitary matrix and $\mathbf{O}$ is the $N \times M$ null matrix. The expressions for the other matrices that compose the eigenvalue problem are

$$
\begin{aligned}
\mathbf{D} & =\operatorname{diag}\left(h_{1}^{-2}, h_{2}^{-2}, \ldots, h_{M}^{-2}\right) \\
C_{i j} & =\frac{\partial^{2} g\left(\mathbf{s}_{i}, \mathbf{s}_{j}\right)}{\partial l \partial l^{\prime}}, \quad i \neq j \\
C_{i i} & =-\frac{1}{\omega_{i}} \sum_{\substack{j=1 \\
j \neq i}}^{N} \omega_{j} \frac{\partial^{2} g\left(\mathbf{s}_{i}, \mathbf{s}_{j}\right)}{\partial l \partial l^{\prime}} \\
L_{i j} & =\widehat{\mathbf{t}}\left(\mathbf{s}_{i}\right) \cdot \overline{\mathbf{G}}_{s t}\left(\mathbf{s}_{i}, \mathbf{s}_{j}\right) \cdot \widehat{\mathbf{t}}\left(\mathbf{s}_{j}\right), \quad i \neq j \\
L_{i i} & =\frac{1}{\omega_{i}} \int_{\sigma_{i}} \widehat{\mathbf{t}}\left(\mathbf{s}_{i}\right) \cdot \overline{\mathbf{G}}_{s t}\left(\mathbf{s}_{i}, \mathbf{s}^{\prime}\right) \cdot \widehat{\mathbf{t}}\left(l^{\prime}\right) d l^{\prime} \\
R_{i m} & =\frac{\mathbf{e}_{m}^{\mathrm{TE}}\left(\mathbf{s}_{i}\right) \cdot \widehat{\mathbf{t}}\left(\mathbf{s}_{i}\right)}{h_{m}^{2}}, \quad i, j=1,2, \ldots, N ; \\
& m=1,2, \ldots M
\end{aligned}
$$

The solution of (18) provides as eigenvectors the modal coefficients $\left(a_{m}\right)$ and the amplitudes of the transversal current density in the discrete points of the contour modified by the quadrature weights (i.e., $b_{j}=w_{j} J_{t}\left(\mathbf{s}_{j}\right)$ ), and as eigenvalues the TE cutoff wavenumbers $(k)$ of the arbitrary waveguide.

When computing the $L_{i i}$ elements according to (21b), the singular contribution of the function $\overline{\mathbf{G}}_{s t}$ must be carefully considered. The dyadic Green function is composed of four components, i.e., $\bar{G}_{s t}^{x x}, \bar{G}_{s t}^{x y}, \bar{G}_{s t}^{y x}$, and $\bar{G}_{s t}^{y y}$. Two of them, i.e., $\bar{G}_{s t}^{x y}$ and $\bar{G}_{s t}^{y x}$, do not contribute with any singularity, whereas the singularities introduced by $\bar{G}_{s t}^{x x}$ and $\bar{G}_{s t}^{y y}$ are of the same kind as considered previously for the TM case. Therefore, the same technique proposed before for the rigorous treatment of the singularities can also be followed, but considering in this case the unitary tangent vector $\widehat{t}$ present in (21b). In this case, the singular integrals $L_{i i s}$ are defined as

$$
\begin{aligned}
& L_{i i s}^{x x}=\frac{1}{\omega_{i}} \int_{\sigma_{i}} t_{x}\left(\mathbf{s}_{i}\right) \cdot \bar{G}_{s t}^{x x}\left(\mathbf{s}_{i}, \mathbf{s}^{\prime}\right) \cdot t_{x}\left(l^{\prime}\right) d l^{\prime} \\
& L_{i i s}^{y y}=\frac{1}{\omega_{i}} \int_{\sigma_{i}} t_{y}\left(\mathbf{s}_{i}\right) \cdot \bar{G}_{s t}^{y y}\left(\mathbf{s}_{i}, \mathbf{s}^{\prime}\right) \cdot t_{y}\left(l^{\prime}\right) d l^{\prime}
\end{aligned}
$$

whose an analytical solution for each kind of arc is as follows.

1) Linear Arcs:

$$
\begin{aligned}
L_{i i s}^{x x} & =\xi_{1} \cos ^{2} \theta \\
L_{i i s}^{y y} & =\xi_{1} \sin ^{2} \theta \\
\xi_{1} & =-\frac{1}{4 \pi} \ln \left(\frac{\omega_{i}}{2}\right)-1
\end{aligned}
$$

where $\theta$ is the angle between the linear arc and the $\hat{\mathbf{x}}$-axis.

2) Circular Arcs:

$$
\begin{aligned}
L_{i i s}^{x x} & = \pm \xi_{2} t_{x} \sin \varphi_{m} \\
L_{i i s}^{y y} & = \pm\left(-\xi_{2}\right) t_{y} \cos \varphi_{m} \\
\xi_{2} & =-\frac{1}{2 \pi \Delta \varphi_{i}}\left[\sin \left(\frac{\Delta \varphi_{i}}{2}\right) \ln \left|\frac{\Delta \varphi_{i}}{2}\right|-S i\left(\frac{\Delta \varphi_{i}}{2}\right)\right]
\end{aligned}
$$

where the selection of the \pm sign is related to the direction of the segment, clockwise $(+)$ or counterclockwise $(-)$. Moreover, $t_{x}$ and $t_{y}$ are, respectively, the $x$ and $y$ components of vector $\widehat{\mathbf{t}}\left(\mathbf{s}_{i}\right)$, $\Delta \varphi_{i}=\varphi_{i 2}-\varphi_{i 1}$ and $\varphi_{m}=0.5\left(\varphi_{i 1}+\varphi_{i 2}\right)$ with $\varphi_{i 1}$ and $\varphi_{i 2}$ being the parametric circular initial and final angles for $\sigma_{i}$, as defined in [7]. The definition of the function $S i$ can also be found in [7, eq. (41)].

3) Elliptical Arcs:

$$
\begin{aligned}
L_{i i s}^{x x} & = \pm \xi_{3} t_{x}\left[a \cos \alpha \sin \eta_{m}+b \sin \alpha \cos \eta_{m}\right] \\
L_{i i s}^{y y} & = \pm \xi_{3} t_{y}\left[a \sin \alpha \sin \eta_{m}-b \cos \alpha \cos \eta_{m}\right] \\
\xi_{3} & =-\frac{\left|\Delta \eta_{i}\right|}{2 \pi \Delta \eta_{i}}\left[\sin \left(\frac{\Delta \eta_{i}}{2}\right) \ln \left|\frac{\Delta \eta_{i}}{2}\right|-S i\left(\frac{\Delta \eta_{i}}{2}\right)\right]
\end{aligned}
$$

where the parameterization and notation previously described in Sections II-A.3 and II-B.2 has been used.

\section{Coupling Coefficients}

In order to use this Nyström-based BI-RME method within CAD tools of complex passive waveguide components, an efficient technique for computing the coupling coefficients between two cascaded arbitrarily shaped waveguides is required. To solve this problem, we choose the same standard rectangular contour for the application of the Nyström and BI-RME methods to both arbitrary waveguides. Once the cutoff frequencies are obtained, and after some post-processing explained below, the required coupling coefficients are easily computed by means of the following expression:

$$
\left\langle\mathbf{e}_{i}^{\left(\mathrm{AW}_{1}\right)}, \mathbf{e}_{j}^{\left(\mathrm{AW}_{2}\right)}\right\rangle=\sum_{n=1}^{N_{(\mathrm{RW})}}\left\langle\mathbf{e}_{i}^{\left(\mathrm{AW}_{1}\right)}, \mathbf{e}_{n}^{(\mathrm{RW})}\right\rangle\left\langle\mathbf{e}_{n}^{(\mathrm{RW})}, \mathbf{e}_{j}^{\left(\mathrm{AW}_{2}\right)}\right\rangle
$$


where the $\left\langle\mathbf{e}_{i}^{\left(\mathrm{AW}_{k}\right)}, \mathbf{e}_{n}^{(\mathrm{RW})}\right\rangle$ term represents the coupling integral between the $i$ th mode of the $k$ th arbitrary waveguide and the $n$th mode of the common rectangular waveguide.

To derive the expressions for the required coupling coefficients between each arbitrary waveguide and the auxiliary rectangular contour, the fast method originally proposed in [8] will be adapted to our Nyström-based formulation. Such a set of coupling integrals is defined as follows:

$$
I_{p q}=\int_{S} \mathbf{e}_{p}^{\square} \cdot \mathbf{e}_{q}^{\diamond} d S
$$

where $\mathbf{e}_{p}^{\square}$ and $\mathbf{e}_{q}^{\diamond}$ are, respectively, the normalized electric modal vectors of the rectangular and arbitrarily shaped waveguides.

Starting with the TM case, if the Nyström method is applied to the IE (2), the following expression is directly obtained for the potential scalar of such modes:

$$
\psi_{m}^{\mathrm{TM} \diamond}(\mathbf{r})=k^{\prime} \sum_{i=1}^{N} g\left(\mathbf{r}, \mathbf{s}_{i}\right) b_{i}^{\prime}+k^{\prime} \sum_{m=1}^{M^{\prime}} \frac{\psi_{m}^{\mathrm{TM}}(\mathbf{r})}{h_{m}^{\prime 2}} a_{m}^{\prime} .
$$

This expression leads to normalized vector mode function for the TM modes if the modal expansion coefficients $a_{m}^{\prime}$ satisfy that $\mathbf{a}^{T} \mathbf{a}^{\prime}=k^{\prime 2}$ (see justification in Appendix II).

Therefore, the normalized transversal electric field for the TM modes can finally be obtained as follows:

$$
\begin{aligned}
\mathbf{e}_{m}^{\mathrm{TM} \diamond}(\mathbf{r}) & =\frac{-\nabla_{T} \psi_{m}^{\mathrm{TM} \diamond}(\mathbf{r})}{k^{\prime}} \\
& =-\sum_{i=1}^{N} \nabla_{T} g\left(\mathbf{r}, \mathbf{s}_{i}\right) b_{i}^{\prime}+\sum_{m=1}^{M^{\prime}} \frac{\mathbf{e}_{m}^{\mathrm{TM} \square}(\mathbf{r})}{h_{m}^{\prime}} a_{m}^{\prime}
\end{aligned}
$$

where $\nabla_{T}$ is the 2-D nabla operator in the transversal coordinate system and $\mathbf{e}_{m}^{\mathrm{TM}} \square$ is the normalized modal vector of the auxiliary standard rectangular waveguide that must accomplish $\mathbf{e}_{m}^{\mathrm{TM} \square}=-\nabla_{T} \psi_{m}^{\mathrm{TM} \square} / h_{m}^{\prime}$.

For the TE case, we must apply the Nyström method to IE (14), thus obtaining the following expression for the tangent electric field:

$$
\begin{array}{r}
\mathbf{e}_{m}^{\mathrm{TE} \diamond}(\mathbf{r})=\frac{1}{k} \sum_{i=1}^{N} \nabla \frac{\partial g\left(\mathbf{r}, \mathbf{s}_{i}\right)}{\partial l} b_{i}-k \sum_{i=1}^{N} \overline{\mathbf{G}}_{s t}\left(\mathbf{r}, \mathbf{s}_{i}\right) \\
\cdot \widehat{\mathbf{t}}\left(\mathbf{s}_{i}\right) b_{i}-k \sum_{m=1}^{M} \frac{\mathbf{e}_{m}^{\mathrm{TE} \square}(\mathbf{r})}{h_{m}^{2}} a_{m}
\end{array}
$$

which will be adequately normalized if coefficients $a_{m}$ and $b_{i}$ satisfy the condition derived in Appendix II.

Now, using expressions (31) and (32) for the normalized TM and TE modal vectors, the required coupling integrals can finally be written as

$$
I_{p q}^{\mathrm{TM}-\mathrm{TM}}=h_{p}^{\prime} \sum_{j=1}^{N} R_{j p}^{\prime} b_{j}^{\prime q}+\frac{a_{p}^{\prime q}}{h_{p}^{\prime}}
$$

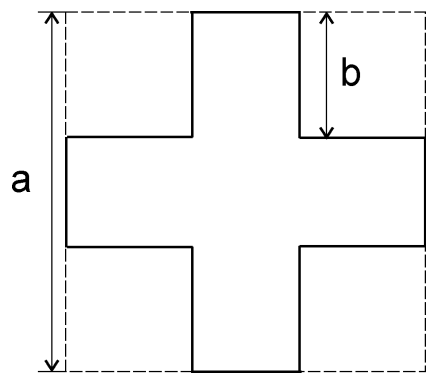

Fig. 2. Geometry of a cross-shaped iris.

$$
\begin{aligned}
& I_{p q}^{\mathrm{TE}-\mathrm{TE}}=k_{q}\left[\sum_{j=1}^{N} R_{j p} b_{j}^{q}+\frac{a_{p}^{q}}{h_{p}^{2}}\right] \\
& I_{p q}^{\mathrm{TM}-\mathrm{TE}}=-\frac{1}{k_{q}} \sum_{j=1}^{N} R_{j p}^{\prime \prime} b_{j}^{q} \\
& I_{p q}^{\mathrm{TE}-\mathrm{TM}}=0
\end{aligned}
$$

where the $R_{j p}^{\prime}$ and $R_{j p}$ terms are the entries of the $\mathbf{R}^{\prime}$ and $\mathbf{R}$ matrices just defined before, and the $a_{p}^{\prime}, b_{j}^{\prime}, a_{p}$, and $b_{j}$ coefficients are the solutions of the Nyström-based TM and TE eigenvalue problems. Therefore, only the new $R_{j p}^{\prime \prime}$ terms are easily evaluated as follows:

$$
R_{j p}^{\prime \prime}=\mathbf{e}_{p}^{\mathrm{TM} \square}\left(\mathbf{s}_{j}\right) \cdot \widehat{\mathbf{t}}\left(\mathbf{s}_{j}\right) .
$$

\section{VALIDATION RESULTS}

Here, we discuss the accuracy and efficiency of the Nyström-based BI-RME approach through several application examples. First, we have computed the complete modal chart of two well-known irises, i.e., a crossed rectangular waveguide and a "triseptum" circular waveguide. Secondly, making use of the novel CAD tool, we have analyzed and designed two complex passive waveguide devices: an inductively coupled rectangular waveguide filter with rounded corners, and a dual-mode filter in circular waveguide technology with elliptical irises and tuning elements. All these examples have been successfully validated through comparisons with numerical and experimental results, which are either available in the technical literature or provided by the authors.

In order to show the gain in efficiency of the new modal method proposed in this paper, CPU computation times for the considered examples are being compared with those obtained applying the traditional Galerkin-based BI-RME approach. All CPU costs offered here have been obtained with a Pentium IV platform at $2.4 \mathrm{GHz}$ with 1-GB RAM.

\section{A. Cross-Shaped Iris}

First, we have considered the cross-shaped iris whose geometry is shown in Fig. 2. These irises are typically used as inter-cavity coupling elements in circular waveguide dual-mode filters (see, for instance, [19]).

Making use of the Nyström-based BI-RME method, we have computed the normalized cutoff frequencies as a function of $b / a$. In Fig. 3, the results predicted by our method (with solid 


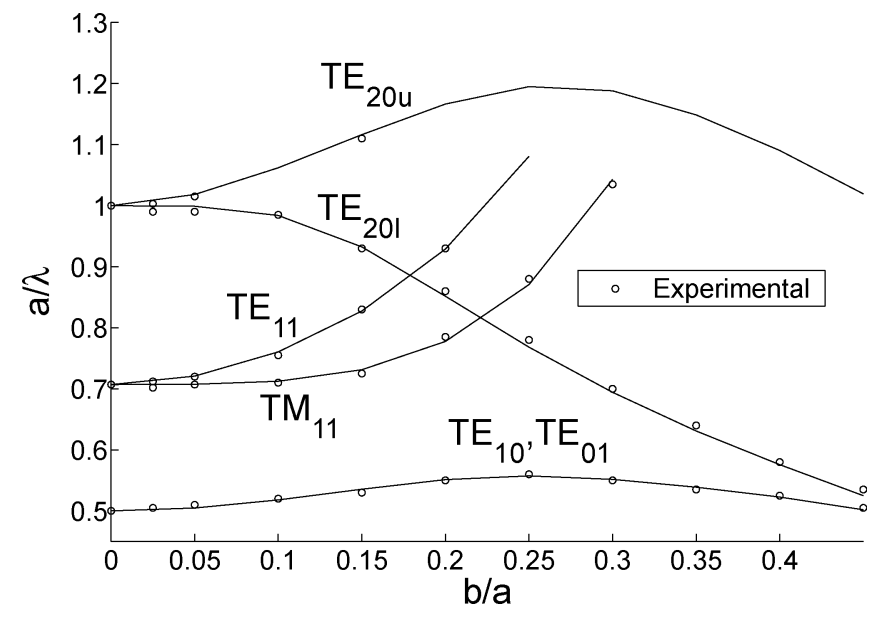

Fig. 3. Normalized cutoff frequencies as function of $b / a$ for the cross-shaped iris shown in Fig. 2.

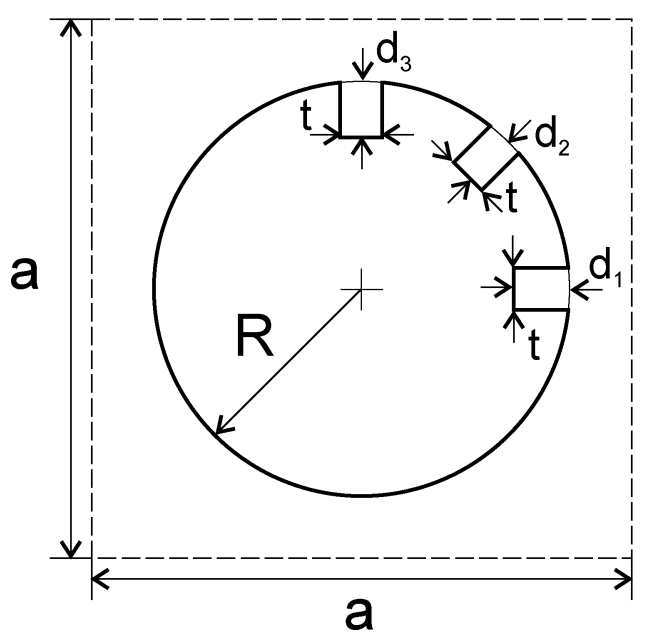

Fig. 4. Circular iris with three rectangular metallic insertions $\left(d_{1}=1.64 \mathrm{~mm}\right.$, $d_{2}=2.72 \mathrm{~mm}, d_{3}=1.74 \mathrm{~mm}, t=2 \mathrm{~mm}, R=12 \mathrm{~mm}$, and $a=25 \mathrm{~mm}$ ).

lines) are successfully compared with experimental data from [20] (with points). For labeling the modes, the same nomenclature used in [20] has been followed.

\section{B. Ridged Circular Iris}

The next example deals with the modal analysis of a circular waveguide with three metallic insertions (the "triseptum" waveguide) shown in Fig. 4, which is typically used in circular waveguide dual-mode filters. The dimensions of this example have been selected from a real device operating at $12 \mathrm{GHz}$ [21].

Using the Nyström-based BI-RME formulation, the first 75 modes have been computed using 500 modes of the auxiliary square waveguide shown in Fig. 4. Table I shows the ten lower cutoff frequencies, and their relative error when compared with the Galerkin implementation [7]. The analysis of the accuracy of the computed frequencies reveals that the mean value of the relative error is $0.2 \%$. The CPU time required for computing the modal spectrum has been of $5.5 \mathrm{~s}$ using the Nyström method, while the Galerkin technique takes $32.4 \mathrm{~s}$, which represents an overall reduction of $83 \%$.

The first coupling coefficients between the "triseptum" waveguide and a circular waveguide of diameter equal to $24 \mathrm{~mm}$
TABLE I

CUTOFF FREQUENCIES FOR THE “TRISEPTUM” WAVEGUIDE

\begin{tabular}{c|c||c|c}
\hline Order & $\begin{array}{c}\text { Mode Type } \\
\text { (TE/TM) }\end{array}$ & $\begin{array}{c}\text { Nyström } \\
(\mathrm{GHz})\end{array}$ & $\begin{array}{c}\text { Relative } \\
\text { Error }(\%)\end{array}$ \\
\hline 1 & TE & 7.16415 & 0.09 \\
\hline 2 & TE & 7.42636 & 0.11 \\
\hline 3 & TM & 9.97961 & 0.13 \\
\hline 4 & TE & 11.7858 & 0.29 \\
\hline 5 & TE & 11.8576 & 0.21 \\
\hline 6 & TE & 15.0568 & 0.37 \\
\hline 7 & TE & 15.3597 & 0.04 \\
\hline 8 & TM & 15.5577 & 0.13 \\
\hline 9 & TM & 16.1950 & 0.12 \\
\hline 10 & TE & 16.6051 & 0.36 \\
\hline
\end{tabular}

TABLE II

COUPLING COEFFICIENTS BETWEEN THE "TRISEPTUM" WAVEGUIDE AND THE CIRCULAR ONE

\begin{tabular}{l|l|l|l|l}
\hline & \multicolumn{4}{|c}{ "Triseptum" waveguide modes } \\
\cline { 2 - 5 } & \multicolumn{1}{|c|}{$T E_{1}$} & \multicolumn{1}{|c}{$T E_{2}$} & $T M_{1}$ & $T E_{3}$ \\
\hline \hline$T E_{11 s}$ & 0.68196 & 0.69371 & -- & -0.04683 \\
& $9 \cdot 10^{-4}$ & $5.1 \cdot 10^{-3}$ & & $1.1 \cdot 10^{-3}$ \\
\hline$T E_{11 c}$ & -0.67994 & 0.69601 & -- & 0.05407 \\
& $4.5 \cdot 10^{-3}$ & $4 \cdot 10^{-4}$ & & $1 \cdot 10^{-3}$ \\
\hline$T M_{01}$ & 0.07117 & 0.00008 & -0.93487 & 0.06039 \\
& $1.5 \cdot 10^{-3}$ & $1 \cdot 10^{-6}$ & $1.8 \cdot 10^{-3}$ & $1.2 \cdot 10^{-3}$ \\
\hline$T E_{21 s}$ & 0.00298 & 0.00062 & -- & 0.89631 \\
& $7 \cdot 10^{-4}$ & $1 \cdot 10^{-5}$ & & $3.9 \cdot 10^{-3}$ \\
\hline$T E_{21 c}$ & 0.00062 & -0.00759 & -- & -0.09502 \\
& $4.8 \cdot 10^{-5}$ & $1.2 \cdot 10^{-3}$ & & $1.3 \cdot 10^{-2}$ \\
\hline
\end{tabular}

are collected in Table II. The absolute error between such Nyström-based results and the Galerkin ones [7] have also been included in Table II and are denoted by italic letters.

\section{Inductively Coupled Rectangular Waveguide Filter With Rounded Corners}

For evaluating the new CAD tool, we have first chosen an inductively coupled rectangular waveguide filter with rounded corners (see geometry and dimensions in Fig. 5) originally designed for operation at $11 \mathrm{GHz}$ with a bandwidth of $300 \mathrm{MHz}$ in [22]. This structure is composed of the cascaded connection of two kinds of transitions: one between rectangular waveguides and rounded coupling irises, and another one between rectangular waveguides with rounded corners. Therefore, the full-wave analysis of this structure will allow to validate the new theoretical aspects described in Section II-C.

For verification purposes, a prototype of this filter has been manufactured (see Fig. 6). The simulated scattering parameters of such a structure are shown in Fig. 7, where they are successfully compared with the experimental results of the manufactured prototype. Such results were obtained using 15 accessible modes, 70 basis functions, and 250 kernel terms in the IEs related to the solution of each discontinuity. These simulating parameters involved a total CPU effort of $204 \mathrm{~s}$ (1000 frequency points) for the Galerkin method, and $144 \mathrm{~s}$ 


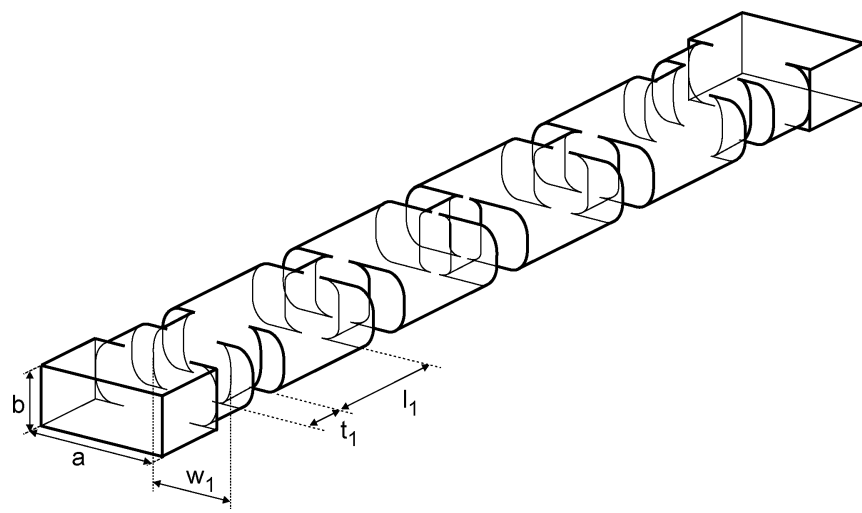

Fig. 5. Inductive filter with rounded corners. The dimensions are: $a=22.86 \mathrm{~mm}, b=10.16 \mathrm{~mm}, l_{1}=4.00 \mathrm{~mm}, l_{2}=14.29 \mathrm{~mm}$, $l_{3}=15.84 \mathrm{~mm}, t_{1}=1.70 \mathrm{~mm}, t_{2}=1.77 \mathrm{~mm}, t_{3}=1.78 \mathrm{~mm}$, $w_{1}=10.50 \mathrm{~mm}, w_{2}=6.70 \mathrm{~mm}$, and $w_{3}=6.15 \mathrm{~mm}$. The radius of the rounded corners is $R=2.00 \mathrm{~mm}$.

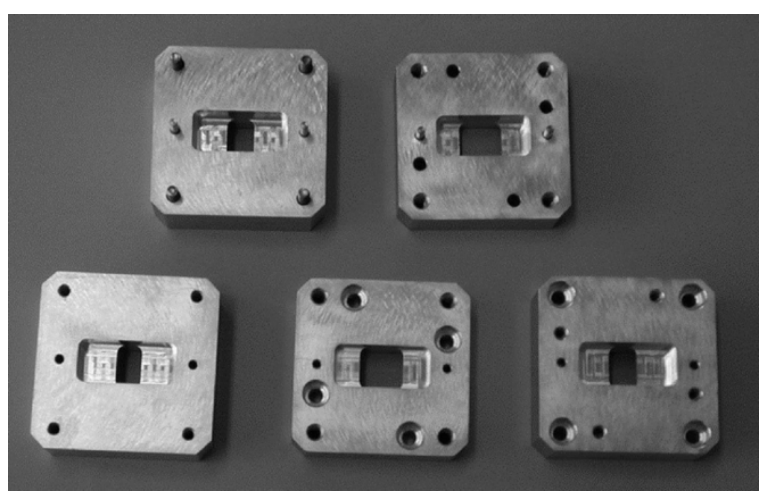

Fig. 6. Internal pieces of the inductive filter with rounded corners. Each one is composed of two half-cavities and a coupling iris.

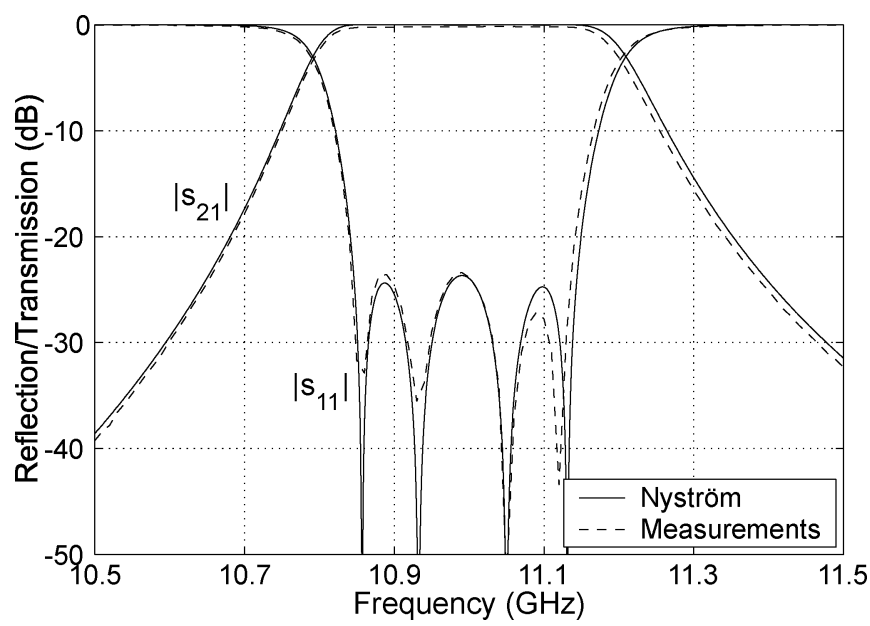

Fig. 7. Magnitude of the reflection $\left(S_{11}\right)$ and transmission $\left(S_{21}\right)$ coefficients of the inductively coupled rectangular waveguide filter with rounded corners shown in Fig. 5. Solid line: authors' results. Dashed lines: authors' measurements of the manufactured prototype (see Fig. 6).

for the new Nyström-based approach. In this example, the total gain in computational cost is approximately $30 \%$, which clearly validates the Nyström method as a good alternative to the Galerkin approach for providing accurate results in quite lower CPU times.

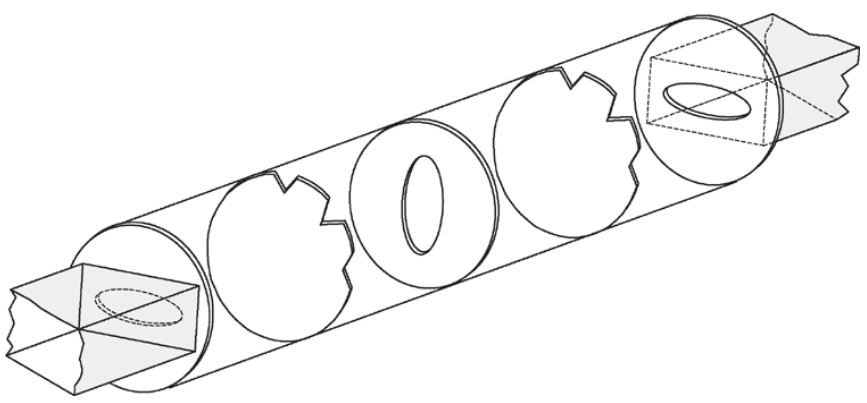

Fig. 8. Geometry of the four-pole dual-mode filter in circular waveguide technology with elliptical irises and tuning elements.

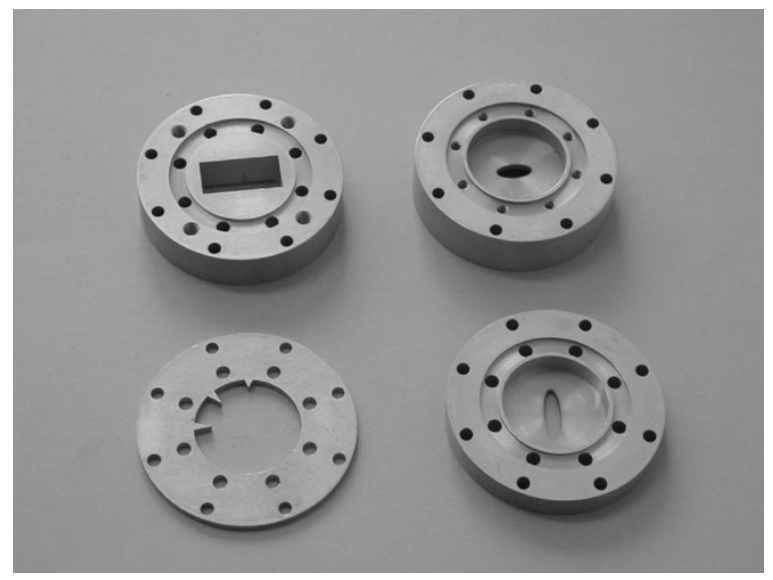

Fig. 9. Internal pieces of the dual-mode circular waveguide filter with elliptical irises and tuning elements.

\section{Circular Waveguide Dual-Mode Filter With Elliptical Irises and Tuning Elements}

After validating the new developed CAD tool based on the Nyström method, we have used such a tool for the design of a four-pole dual-mode filter in circular waveguide technology. As can be seen in Fig. 8, such a structure is basically composed of two circular waveguide cavities coupled through a rotated elliptical iris and fed to the input/output waveguides by means of two elliptical irises. In order to tune and couple each pair of degenerated modes, in the middle plane of each cavity we have placed a short circular waveguide with three metallic insertions (see Fig. 8).

In order to verify the geometrical dimensions provided by the novel CAD tool, we have manufactured a prototype of the circular waveguide dual-mode filter just described. This filter is composed of several pieces containing the input/output waveguides, the irises, and the resonators of the structure (see the photograph shown in Fig. 9), which are connected in cascade to build the whole filter.

The simulated reflection and transmission coefficients of this filter are compared with the authors' measurements in Fig. 10. Some slight misalignments can be observed between both results in the bandpass frequencies, which can be attributed to manufacturing tolerances. Nevertheless, as usually happens with these very sensitive structures, the desired electrical response could be recovered by replacing the fixed triangular tuning elements by real adjustable screws. 


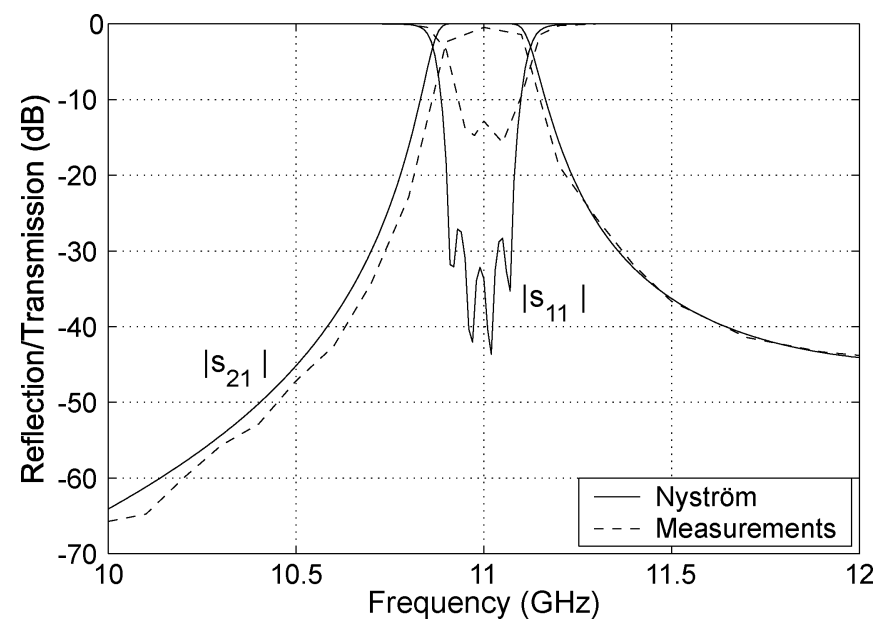

Fig. 10. Magnitude of the reflection $\left(S_{11}\right)$ and transmission $\left(S_{21}\right)$ coefficients of the dual-mode circular waveguide filter with elliptical irises and tuning elements shown in Fig. 8. Solid line: authors' results. Dashed lines: authors' measurements of the manufactured prototype (see Fig. 9).

During the CAD stages of this dual-mode filter, it was required to use 100 accessible modes, 300 basis functions, and 900 kernel terms in the corresponding IEs for obtaining enough accurate and convergent results. The computational effort related to each simulation of the whole structure has been of $3.5 \mathrm{~s}$ per frequency point, which was rather adequate for design purposes.

\section{CONCLUSIONS}

A fast and rather accurate Nyström-based BI-RME method has been applied to the complete CAD of complex passive devices composed of cascaded arbitrarily shaped waveguides defined by linear, circular, and elliptical arcs. This novel method offers some advantages compared to the standard Galerkin BI-RME approach: the first one is the simplicity of the implementation, and the second one is the reduction of the computational time. The new developed method has been successfully verified through several application examples of great practical interest such as the modal chart computation of cross-shaped and ridged circular irises, the efficient full-wave analysis of inductively coupled rectangular waveguide filters with rounded corners, and the complete CAD of circular waveguide dual-mode filters with elliptical irises and tuning elements. CPU times have been included to validate the efficiency improvement provided by the inclusion of the Nyström approach within modern CAD tools.

\section{APPENDIX I}

\section{DOUble DERIVATIVE OF THE SCALAR 2-D GREEN FunCTION}

When solving the TE modes in the BI-RME method following the Nyström approach, the evaluation of $\partial^{2} g / \partial l^{\prime} \partial l$ is required [see (20)], where $g$ is the scalar Green function. For that purpose, a rapidly convergent expression for the dyadic $\nabla \nabla^{\prime} g$ has been derived from the bilinear form of the scalar 2-D Green function [23]. The dyadic $\nabla \nabla^{\prime} g$ has been accelerated using the Poisson summation formula [24]. The directional derivatives have been then computed as follows:

$$
\frac{\partial g}{\partial l}=\nabla g \cdot \hat{\mathbf{t}} \Rightarrow \frac{\partial^{2} g}{\partial l \partial l^{\prime}}=\hat{\mathbf{t}} \cdot \nabla \nabla^{\prime} g \cdot \hat{\mathbf{t}}^{\prime}
$$

where

$$
\nabla \nabla^{\prime} g=\frac{\partial^{2} g}{\partial x \partial x^{\prime}} \hat{\mathbf{x}} \hat{\mathbf{x}}+\frac{\partial^{2} g}{\partial x \partial y^{\prime}} \hat{\mathbf{x}} \hat{\mathbf{y}}+\frac{\partial^{2} g}{\partial y \partial x^{\prime}} \hat{\mathbf{y}} \hat{\mathbf{x}}+\frac{\partial^{2} g}{\partial y \partial y^{\prime}} \hat{\mathbf{y}} \hat{\mathbf{y}}
$$

being each term a component of the dyadic [25].

For each component of the dyadic $\nabla \nabla^{\prime} g$, a corresponding rapidly convergent expression, which is going to be presented below, has been obtained.

\section{A. Component $\hat{\mathbf{x}} \hat{\mathbf{x}}$}

$$
\frac{\partial^{2} g}{\partial x \partial x^{\prime}}=\frac{\pi}{4 a^{2}} \sum_{n=-\infty}^{\infty} T_{n}^{10}-T_{n}^{11}+T_{n}^{00}-T_{n}^{01}
$$

where

$$
\begin{aligned}
T_{n}^{p q} & =\frac{\cosh \left[\frac{\pi}{a}\left(\rho_{n}^{q}\right)\right] \cos \left[\frac{\pi}{a}\left(\gamma_{p}\right)\right]-1}{\left\{\cosh \left[\frac{\pi}{a}\left(\rho_{n}^{q}\right)\right]-\cos \left[\frac{\pi}{a}\left(\gamma_{p}\right)\right]\right\}^{2}} \\
\gamma_{p} & =x-(-1)^{p} x^{\prime} \\
\rho_{n}^{q} & =y-(-1)^{q} y^{\prime}+2 n b .
\end{aligned}
$$

\section{B. Component $\hat{\mathbf{x}} \hat{\mathbf{y}}$}

$$
\frac{\partial^{2} g}{\partial x \partial y^{\prime}}=\frac{\pi}{4 a^{2}} \sum_{n=-\infty}^{\infty} T_{n}^{11}+T_{n}^{10}-T_{n}^{01}-T_{n}^{00}
$$

with

$$
\begin{aligned}
T_{n}^{p q} & =S_{n}^{q} \frac{\sin \left[\frac{\pi}{a}\left(\gamma_{p}\right)\right] \sinh \left[\frac{\pi}{a}\left|\rho_{n}^{q}\right|\right]}{\left\{\cosh \left[\frac{\pi}{a}\left|\rho_{n}^{q}\right|\right]-\cos \left[\frac{\pi}{a}\left(\gamma_{p}\right)\right]\right\}^{2}} \\
S_{n}^{q} & =\operatorname{sgn}\left[\frac{\pi}{b}\left(y-(-1)^{q} y^{\prime}\right)+2 n \pi\right] .
\end{aligned}
$$

\section{Components $\hat{\mathbf{y}} \hat{\mathbf{x}}, \hat{\mathbf{y}} \hat{\mathbf{y}}$}

The remaining components can be obtained from the previous ones by just using symmetry properties of the Green function

$$
\begin{aligned}
\frac{\partial^{2} g}{\partial y \partial x^{\prime}}\left(x, y ; x^{\prime}, y^{\prime} ; a, b\right) & =\frac{\partial^{2} g}{\partial x \partial y^{\prime}}\left(y, x ; y^{\prime}, x^{\prime} ; b, a\right) \\
\frac{\partial^{2} g}{\partial y \partial y^{\prime}}\left(x, y ; x^{\prime}, y^{\prime} ; a, b\right) & =\frac{\partial^{2} g}{\partial x \partial x^{\prime}}\left(y, x ; y^{\prime}, x^{\prime} ; b, a\right) .
\end{aligned}
$$




\section{APPENDIX II \\ NORMALIZATION OF THE EIGENVECTORS}

\section{A. TM Modes}

The scalar potential of the TM modes of the arbitrarily shaped waveguide must be normalized according to

$$
\int_{\Omega}\left(\psi^{\mathrm{TM} \diamond}(\mathbf{r})\right)^{2} d \Omega=1
$$

This normalization condition can be developed using the expression for $\psi^{\mathrm{TM} \diamond}(\mathbf{r})$ derived from (2), thus obtaining

$$
\begin{aligned}
& k^{\prime 2} \int_{\Omega} \int_{\sigma} \int_{\sigma} g\left(\mathbf{r}, \mathbf{s}^{\prime}\right) g\left(\mathbf{r}, \mathbf{s}^{\prime \prime}\right) J_{z}\left(l^{\prime}\right) J_{z}\left(l^{\prime \prime}\right) d l^{\prime} d l^{\prime \prime} d \Omega \\
& +k^{\prime 2} \int_{\Omega} \sum_{m=1}^{M^{\prime}} \sum_{n=1}^{M^{\prime}} \frac{\psi_{m}^{\mathrm{TM} \square}(\mathbf{r}) \psi_{n}^{\mathrm{TM} \square}(\mathbf{r})}{h_{m}^{\prime 2} h_{n}^{\prime 2}} a_{m}^{\prime} a_{n}^{\prime} d \Omega \\
& +2 k^{\prime 2} \int_{\Omega} g\left(\mathbf{r}, \mathbf{s}^{\prime}\right) J_{z}\left(l^{\prime}\right) \sum_{m=1}^{M^{\prime}} \frac{\psi_{m}^{\mathrm{TM}}(\mathbf{r})}{h_{m}^{\prime 2}} a_{m}^{\prime} d l^{\prime} d \Omega=1 .
\end{aligned}
$$

If we consider the following eigenfunction expansion of the Green function:

$$
g(\mathbf{r}, \mathbf{s})=\sum_{i} \frac{\psi_{i}^{\mathrm{TM} \square}(\mathbf{r}) \psi_{i}^{\mathrm{TM} \square}(\mathbf{s})}{h_{i}^{\prime 2}}
$$

and taking into account that the scalar potentials of the rectangular waveguide are also normalized according to

$$
\int_{\Omega} \psi_{m}^{\mathrm{TM} \square}(\mathbf{r}) \psi_{n}^{\mathrm{TM} \square}(\mathbf{r}) d \Omega=\delta_{m n}
$$

(46) can be rewritten as follows:

$$
\begin{aligned}
1=k^{\prime 2} & \sum_{m=1}^{M^{\prime}} \int_{\sigma} \frac{\psi_{m}^{\mathrm{TM}}\left(\mathbf{s}^{\prime}\right)}{h_{m}^{\prime 2}} J_{z}\left(l^{\prime}\right) d l^{\prime} \int_{\sigma} \frac{\psi_{m}^{\mathrm{TM} \square}\left(\mathbf{s}^{\prime \prime}\right)}{h_{m}^{\prime 2}} J_{z}\left(l^{\prime \prime}\right) d l^{\prime \prime} \\
& +k^{\prime 2} \sum_{m=1}^{M^{\prime}} \frac{a_{m}^{\prime 2}}{h_{m}^{\prime 4}}+2 k^{\prime 2} \sum_{m=1}^{M^{\prime}} \frac{a_{m}^{\prime}}{h_{m}^{\prime 2}} \int_{\sigma} \frac{\psi_{m}^{\mathrm{TM} \square}\left(\mathbf{s}^{\prime}\right)}{h_{m}^{\prime 2}} J_{z}\left(l^{\prime}\right) d l^{\prime} .
\end{aligned}
$$

Now applying the Nyström technique and making use of the definitions of submatrices collected in (6)-(8), (49) can be written in matrix form as

$$
k^{\prime 2}\left[\mathbf{b}^{\prime T} \mathbf{R}^{\prime} \mathbf{R}^{\prime T} \mathbf{b}^{\prime}+\mathbf{a}^{\prime T} \mathbf{D}^{\prime T} \mathbf{D}^{\prime} \mathbf{a}^{\prime}+2 \mathbf{b}^{\prime T} \mathbf{R}^{\prime} \mathbf{D}^{\prime} \mathbf{a}^{\prime}\right]=1
$$

Finally, if we employ (5) in (50), the normalization condition is established as

$$
\mathbf{a}^{\prime T} \mathbf{a}^{\prime}=k^{\prime 2}
$$

\section{B. TE Modes}

In the case of the TE modes of the arbitrarily shaped waveguide, the electric modal vector must be normalized according to

$$
\int_{\Omega} \mathbf{e}^{\mathrm{TE} \diamond}(\mathbf{r}) \cdot \mathbf{e}^{\mathrm{TE} \diamond}(\mathbf{r}) d \Omega=1
$$

In this case, we must proceed in the same way proposed earlier for the TM normalization. First, we insert the definition of $\mathbf{e}^{\mathrm{TE} \diamond}(\mathbf{r})$ given by (14) within (52). Next, the following eigenfunction expansion for the dyadic Green function $\overline{\mathbf{G}}_{s t}$ is considered:

$$
\overline{\mathbf{G}}_{s t}(\mathbf{r}, \mathbf{s})=\sum_{i} \frac{\mathbf{e}_{i}^{\mathrm{TE} \square}(\mathbf{r}) \mathbf{e}_{i}^{\mathrm{TE} \square}(\mathbf{s})}{h_{i}^{2}}
$$

and the following condition for the modal vectors of the rectangular waveguide is used:

$$
\int_{\Omega} \mathbf{e}_{m}^{\mathrm{TM} \square}(\mathbf{r}) \mathbf{e}_{n}^{\mathrm{TE} \square}(\mathbf{r}) d \Omega=0
$$

The following normalization condition for the eigenvector solutions of the problem defined by (18) is then finally obtained:

$$
\begin{aligned}
\mathbf{x}^{T} \mathbf{B} \mathbf{x} & =1 \\
\mathbf{x}^{T} \mathbf{A} \mathbf{x} & =k^{2}
\end{aligned}
$$

where $\mathbf{x}, \mathbf{B}$, and $\mathbf{A}$ result in expressing the cited eigenvalue problem (18) in compact form $\left[\mathbf{A}-k^{2} \mathbf{B}\right] \mathbf{x}=0$.

\section{ACKNOWLEDGMENT}

The authors would like to thank Prof. G. Conciauro, University of Pavia, Pavia, Italy, for his valuable comments concerning the normalization issues discussed in Appendix II, and Dr. M. Guglielmi, European Space Research and Technology Centre (ESTEC), European Space Agency (ESA), Noordwijk, The Netherlands, for providing the prototypes used for verification purposes.

\section{REFERENCES}

[1] J. Uher, J. Bornemann, and U. Rosenberg, Waveguide Components for Antenna Feed Systems: Theory and CAD. Norwood, MA: Artech House, 1993.

[2] P. P. Silvester and G. Pelosi, Finite Elements for Wave Electromagnetics. Piscataway, NJ: IEEE Press, 1994.

[3] A. Taflove, Computational Electromagnetics: The Finite-Difference Time-Domain Method. Norwood, MA: Artech House, 1995.

[4] T. Itoh, Numerical Techniques for Microwave and Millimeter-Wave Passive Structures. New York: Wiley, 1989.

[5] G. Conciauro, M. Guglielmi, and R. Sorrentino, Advanced Modal Analysis-CAD Techniques for Waveguide Components and Filters. Chichester, U.K.: Wiley, 2000.

[6] G. Conciauro, M. Bressan, and C. Zuffada, "Waveguide modes via an integral equation leading to a linear matrix eigenvalue problem," IEEE Trans. Microw. Theory Tech., vol. 32, no. 11, pp. 1495-1504, Nov. 1984. 
[7] S. Cogollos, S. Marini, V. Boria, P. Soto, A. Vidal, H. Esteban, J. V. Morro, and B. Gimeno, "Efficient modal analysis of arbitrarily shaped waveguides composed of linear, circular and elliptical arcs using the BI-RME method," IEEE Trans. Microw. Theory Tech., vol. 51, no. 12, pp. 2378-2390, Dec. 2003.

[8] P. Arcioni, "Fast evaluation of modal coupling coefficients of waveguide step discntinuities," IEEE Microw. Guided Wave Lett., vol. 6, no. 6, pp. 232-234, Jun. 1996.

[9] M. Bozzi, G. Conciauro, and L. Perregrini, "On the evaluation of modal coupling coefficients by contour integrals," IEEE Trans. Microw. Theory Tech., vol. 50, no. 7, pp. 1853-1855, Jul. 2002.

[10] R. F. Harrington, Field Computation by Moment Methods. New York: IEEE Press, 1992.

[11] L. Delves and J. Mohamed, Computational Methods for Integral Equations, 2nd ed. Cambridge, U.K.: Cambridge Univ. Press, 1992.

[12] P. J. Davis and P. Rabinowitz, Methods of Numerical Integration, 2nd ed. San Diego, CA: Academic, 1984.

[13] J. Ottusch and M. Wandzura, "High-order Nyström method for computing waveguide modes," ACES J., vol. 17, no. 1, pp. 84-92, Mar. 2002.

[14] M. Taroncher, A. Vidal, V. E. Boria, S. Marini, P. Soto, S. Cogollos, and B. Gimeno, "Efficient full wave modal analysis of arbitrarily shaped waveguides using BI-RME and Nystrom methods," in Proc. 33rd Eur. Microwave Conf., Munich, Germany, Oct. 2003, pp. 455-458.

[15] M. Taroncher, A. Vidal, V. E. Boria, S. Marini, S. Cogollos, J. Gil, and B. Gimeno, "Efficient CAD tool of complex passive devices composed of arbitrarily shaped waveguides using Nyström and BI-RME methods," in Proc. 34th Eur. Microwave Conf., Amsterdam, The Netherlands, Oct. 2004, pp. 1237-1240.

[16] G. Gerini, M. Guglielmi, and G. Lastoria, "Efficient integral equation formulations for impedance or admittance representation of planar waveguide junction," in IEEE MTT-S Int. Microwave Symp. Dig., Baltimore, MD, Jun. 1998, pp. 1747-1750.

[17] B. Alpert, B. Beylkin, R. Coifman, and V. Rohklin, "Wavelet-like bases for the fast solution of second-kind integral equations," SIAM J. Sci. Comput., vol. 14, no. 1, pp. 159-184, Jan. 1993.

[18] J. J. H. Wang, Generalized Moment Methods in Electromagnetics: Formulation and Computer Solution of Integral Equations. New York: Wiley, 1991.

[19] P. Couffignal, H. Baudrand, and B. Théron, "A new rigorous method for the determination of iris dimensions in dual-mode cavity filters," IEEE Trans. Microw. Theory Tech., vol. 42, no. 7, pp. 1314-1320, Jul. 1994.

[20] H. Stalzer, M. Greenman, and F. Willwerth, "Modes of crossed rectangular waveguide," IEEE Trans. Antennas Propag., vol. 24, no. 2, pp. 220-223, Mar. 1976.

[21] J. R. Montejo-Garai and J. Zapata, "Full-wave design and realization of multicoupled dual-mode circular waveguide filters," IEEE Trans. Microw. Theory Tech., vol. 43, no. 6, pp. 1290-1297, Jun. 1995.

[22] S. Cogollos, V. E. Boria, P. Soto, B. Gimeno, and M. Guglielmi, "Efficient CAD tool for inductively coupled rectangular waveguide filters with rounded corners," in Proc. 31st Eur. Microwave Conf., London, U.K., Sep. 2001, pp. 315-318.

[23] C. A. Balanis, Advanced Engineering Electromagnetics. New York: Wiley, 1989.

[24] P. Morse and H. Feshbach, Methods of Theoretical Physics. New York: McGraw-Hill, 1978.

[25] C.-T. Tai, Dyadic Green Functions in Electromagnetic Theory, 2nd ed, ser. Electromagn. Waves. New York: IEEE Press, 1994.

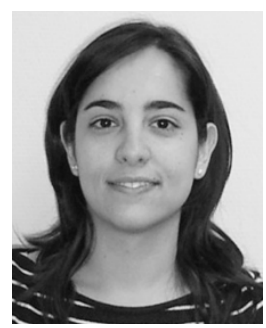

Máriam Taroncher (S'03) was born in Lliria, Valencia, Spain, on October 8, 1979. She received the Telecommunications Engineering degree from the Universidad Politécnica de Valencia (UPV), Valencia, Spain, in 2003, and is currently working toward the Ph.D. degree at UPV.

From 2002 to 2004, she was a Fellow Researcher with the UPV. Since 2004, she has been a Technical Researcher in charge of the experimental laboratory for high power effects in waveguide devices at the Research Institute iTEAM, UPV. Her current research interests include numerical methods for the analysis of waveguide structures and the acceleration of the electromagnetic analysis methods using wavelets.

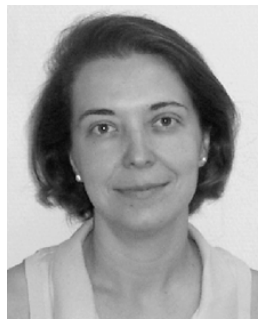

Ana Vidal (M'01) was born in Valencia, Spain in 1970. She received the Ingeniero de Telecomunicación degree from the Universidad Politécnica de Valencia, Valencia, Spain, in 1993.

In 1993, she spent one year with the University of Strathclyde, Glasgow, U.K., under the Erasmus international exchange program. In 1993, she was involved in broad-band communications development with the main research center of Telecom Portugal. She then became a Research Assistant with the Universidad Politécnica de Valencia. In 1995 and 1996, she held a Spanish Trainee position with the European Space research and Technology Centre (ESTEC)-European Space Agency (ESA), Noordwijk, the Netherlands, where she was involved in the study and implementation of software for synthetic aperture radar (SAR) image processing. In 1996, she returned to the Universidad Politécnica de Valencia, where she held several lecturing positions and, in 2001, became an Associate Professor. Her current interests are SAR data processing, SAR speckle noise reduction, and numerical methods for microwave structures analysis including the wavelet transform.

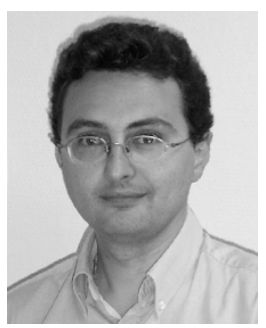

Vicente E. Boria-Esbert (S'91-A'99-SM'02) was born in Valencia, Spain, on May 18, 1970. He received the Ingeniero de Telecomunicación degree (with first-class honors) and Doctor Ingeniero de Telecomunicación degree from the Universidad Politécnica de Valencia, Valencia, Spain, in 1993 and 1997, respectively.

In 1993 he joined the Departamento de Comunicaciones, Universidad Politécnica de Valencia, where he has been an Assistant Lecturer (1993-1995), Lecturer (1996-1997), Associate Professor (1998-2002), and Full Professor (since 2003). In 1995 and 1996, he held a Spanish Trainee position with the European Space Research and Technology Centre (ESTEC)-European Space Agency (ESA), Noordwijk, The Netherlands, where he was involved in the area of electromagnetic (EM) analysis and design of passive waveguide devices. He has authored or coauthored several chapters in technical textbooks, 25 papers in refereed international technical journals, and over 100 papers in international conference proceedings. His current research interests include numerical methods for the analysis of waveguide and scattering structures, automated design of waveguide components, radiating systems, measurement techniques, and power effects in passive waveguide systems. Since 2003, he has served on the Editorial Boards of the Proceedings of the IEE (Microwaves, Antennas, and Propagation) and Radio Science.

Dr. Boria-Esbert is a member of the IEEE Microwave Theory and Techniques Society (IEEE MTT-S) and the IEEE Antennas and Propagation Society (IEEE AP-S) since 1992. Since 2003, he has served on the Editorial Board of the IEEE TRANSACTIONS ON MICROWAVE THEORY AND TECHNIQUES. He is also member of the Technical Committees of the IEEE-MTT International Microwave Symposium and of the European Microwave Conference. He was the recipient of the 1993 Spanish Ministerio de Educación y Ciencia and the 1993 First National Prize of Telecommunication Engineering Studies for his outstanding student record. He was also the recipient of the 2001 Social Council of Universidad Politécnica de Valencia First Research Prize for his outstanding activity during 1995-2000.

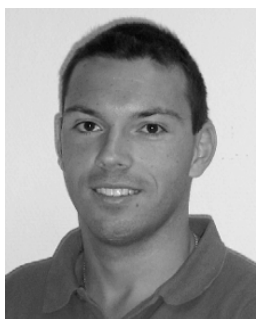

Stephan Marini was born in Cagli, Italy, on January 3, 1976. He received the Laurea degree in electronics engineering from the University of Perugia, Perugia, Italy, in 2001, and is currently working toward the $\mathrm{Ph} . \mathrm{D}$. degree in telecommunications at the Universidad Politécnica de Valencia, Valencia, Spain.

In June 2001, he joined the Departamento de Comunicaciones, Universidad Politécnica de Valencia. His current research interests include numerical methods for the analysis of arbitrary-shaped waveguide and scattering structures. 


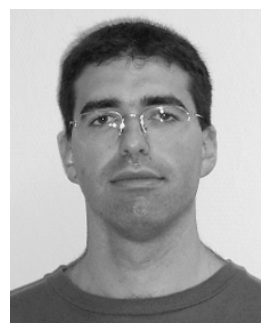

Santiago Cogollos was born in Valencia, Spain, on January 15,1972 . He received the Ingeniero de Telecomunicación and Doctor Ingeniero de Telecomunicación degrees from the Universidad Politécnica de Valencia, Valencia, Spain, in 1996 and 2002, respectively.

In 2000, he joined the Departamento de Comunicaciones, Universidad Politécnica de Valencia, where he was an Assistant Lecturer (2000-2001), a Lecturer (2001-2002), and an Associate Professor (since 2002). He has collaborated with the European Space Research and Technology Centre (ESTEC)-European Space Agency (ESA), Noordwijk, The Netherlands, in the development of modal analysis tools for payload systems in satellites. His current research interests include numerical methods for the analysis of waveguide structures and design of waveguide components for space applications.

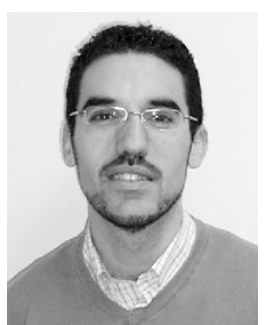

Jordi Gil was born in Valencia, Spain, on April 27, 1977. He received the Licenciado degree in physics from the Universidad de Valencia, in 2000, and is currently working toward the Ph.D. degree at the Universidad Politécnica de Valencia, Valencia, Spain.

From 2001 to 2003, he was with Ingegneria dei Sistemi IDS-S.p.A., Pisa, Italy, where he was involved with the European Union project MMCODEF "Millimeter-wave and Microwave Components Design Framework for Ground and Space Multimedia Network" in collaboration with the European Space Agency (ESA). His current research interests include numerical methods in computer-aided techniques for the analysis and design of microwave passive components such as waveguide structures with dielectric resonators for space applications.

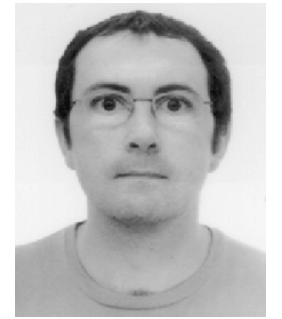

Benito Gimeno (M'01) was born in Valencia, Spain, on January 29, 1964. He received the Licenciado degree in physics and Ph.D. degree from the Universidad de Valencia, Valencia, Spain, in 1987 and 1992, respectively.

From 1987 to 1990, he was a Research Fellow with the Universidad de Valencia. Since 1990, he has been an Assistant Professor with the Departamento de Física Aplicada y Electromagnetismo, Universidad de Valencia, where, in 1997, he became an Associate Professor. From 1994 to 1995, he was a Research Fellow with the European Space Research and Technology Centre (ESTEC)-European Space Agency (ESA). In 2003, he spent three months as a Visiting Scientist with the Università degli Studi di Pavia, Pavia, Italy. His current research interests include the areas of computer-aided techniques for analysis of microwave passive components, waveguide and cavities structures including dielectric resonators, and photonic-bandgap crystals. 